\title{
Effect on louver dampers configuration on gas maldistribution in a heat exchanger for waste heat recovery
}

\author{
Grzegorz Ligus ${ }^{1, *}$, Daniel Zając ${ }^{2}$, and Jatinkumar Panchal $^{3}$ \\ ${ }^{1}$ Faculty of Mechanical Engineering, Opole University of Technology, Opole, Poland \\ ${ }^{2}$ Kelvion Central Europe Sp. z o. o., Opole, Poland \\ ${ }^{3}$ Laser Microjet Solution, Gujarat, India
}

\begin{abstract}
This paper reports the results of visualization research involving a heat exchanger applied for waste heat recovery using of Digital Particle Image Velocimetry (DPIV) method. Five louver dampers configurations responsible for typical operation modes were tested. As a consequence, airflow maldistribution in the tube bundle section and bypass section were identified and described. Based on the measurements and analysis carried out, the authors have developed a way to implement design changes to optimize gas distribution inside the unit.
\end{abstract}

\section{Introduction}

Waste heat recovery unit (WHRU) are found in various process parts such as exhaust gas streams or used drying process air. In the case of process boilers that are commonly applied in the industry practice, WHRUs are called economizers. The task of WHRU is to use the enthalpy of the exhaust gases to heat up another medium and transfer heat into another process area. The application of such equipment leads to an increase in the energy efficiency of the installation in which WHRU is installed [1]. To ensure the required performance in variable operating conditions or balance WHRU with other heat exchange equipment, WHRUs comprise regulating mechanisms in the form of louver dampers of various types and bypass sections. These components are responsible for the distribution of the exhaust gases to individual heat-receiving elements as well as enable complete shutdown of the equipment by initiating bypass flow. Irrespectively of the dimensions of WHRUs, louver dampers are commonly used for regulating. The variations in the configuration of the louver dampers determine the variations in the gas velocity value and its profile [2]. In addition, the location of the louver dampers plays a significant role of pressure drops generation in the apparatus [8]. The total of these changes considerably alters the aerodynamic characteristics of the equipment in relation to the simplifications adopted during the design phase. The objective of the current research involved the development of a correlation between the configuration of louver dampers and variations in the direction and velocity of gas flow in a heat recovery

\footnotetext{
* Corresponding author: g.ligus@po.opole.pl
} 
heat exchanger. Usually, Computational Fluid Dynamics (CFD) is used to evaluate changes of geometrical and flow parameters in industrial equipment $[3,4]$. The idea of reconstructing gas flow patterns as a result of identification of undesired phenomena like vortices or flow maldistribution can provide assistance in redesign industrial apparatus [5]. In the field of experimental research, the DPIV method is mentioned as one of the most excellent tools for assessing gas behaviour in flow devices $[6,7]$.

\section{Experimental setup and methodology}

DPIV method was applied in the experimental research. An industrial scale transparent model of WHRU was built for this purpose. The external dimensions of the device were: length 900 $\mathrm{mm}$, width $456 \mathrm{~mm}$ and height $450 \mathrm{~mm}$. The pitch of the tube arrangement in the bundle was equal to $24 \mathrm{~mm}$. The inlet and outlet with a diameter of $200 \mathrm{~mm}$ were placed in the central section of the front and the back panels. The louver dampers configurations in the WHRU model correspond to three principal modes of operation: full gas flow through the tube bundle section, full gas flow through the bypass section and gas flow separated into two parts: through the tube bundle section and through bypass section, resulting from the selection of an operating mode or controlling of the heat reception intensity. The inclination angles of the louver dampers during transient operation modes were selected to generate pressure drops at similar levels at each stage of opening or closing of both sections. The configuration parameters of the tested louver dampers locations are summarized in Table 1 and their visualizations are given in Figure 1.

Table 1. Louver dampers configuration.

\begin{tabular}{|l|c|c|c|c|}
\hline & $\begin{array}{c}\text { Angle of bypass } \\
\text { louver damper }\end{array}$ & $\begin{array}{c}\text { Angle of main } \\
\text { louver dampers }\end{array}$ & $\begin{array}{c}\text { Pressure drop } \\
\mathbf{\Delta P , ~ P a}\end{array}$ & $\begin{array}{c}\text { Description of the WHRU } \\
\text { operating mode }\end{array}$ \\
\hline Position 1 & $90^{\circ}$ & $0^{\circ}$ & 44 & $\begin{array}{c}\text { Full gas flow through a tube } \\
\text { bundle section }\end{array}$ \\
\hline Position 2 & $70^{\circ}$ & $20^{\circ}$ & 42 & Closing / opening \\
\hline Position 3 & $50^{\circ}$ & $30^{\circ}$ & 42 & Closing / opening \\
\hline Position 4 & $30^{\circ}$ & $40^{\circ}$ & 41 & Closing / opening \\
\hline Position 5 & $0^{\circ}$ & $90^{\circ}$ & 61 & $\begin{array}{c}\text { Full gas flow through a bypass } \\
\text { section }\end{array}$ \\
\hline
\end{tabular}

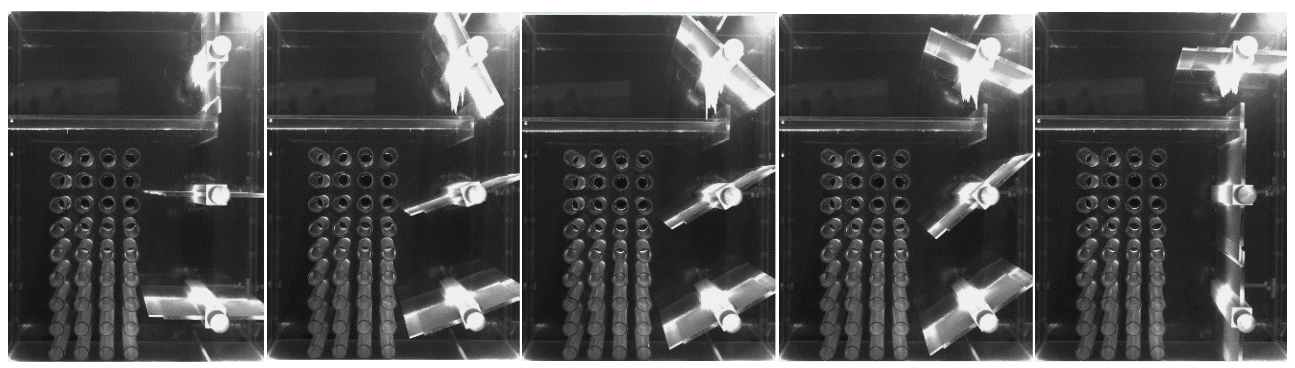

Fig. 1. Visualization of the tested louver dampers configurations.

The study applied flow through WHRU using atmospheric air with a suspended droplets of Di-Ethyl-Hexyl-Sebacat (DEHS) as seeding particles. Airflow was generated by the Helios MVZ315 fan unit. The calculated airflow velocity in the tube bundle section was equal to $4 \mathrm{~m} / \mathrm{s}$ and was within the airflow velocity range recommended by the manufacturer (from 
$2 \mathrm{~m} / \mathrm{s}$ to $6 \mathrm{~m} / \mathrm{s}$ ). The region that was selected for observations was illuminated by application of the laser sheet technique. The laser sheet was generated by the Dantec Dynamics DualPower TR laser and directed with the use of a mirror, to the bottom of the transparent WHRU model, perpendicular to the vertical symmetry line of the inlet. The airflow images (100 images for each measurement series) were recorded using the Dantec Dynamics FlowSense EO 4M camera in the double frame mode. The computer images processing and velocity vector fields analysis were carried out using the Dynamic Studio 2015 software. In a table 2 the technical parameters of the DPIV system were summarized. The pressure drop measurement was performed for each louver dampers configuration regardless of the applied DPIV method. The sketch and the actual view of the experimental stand are presented in Figure 2 and Figure 3.

Table 2. Technical parameters of the DPIV system.

\begin{tabular}{|c|c|}
\hline Parameter & Description \\
\hline Type of the devices & $\begin{array}{c}\text { Camera: Dantec Dynamics FlowSense EO 4M, Laser: Dantec Dynamics } \\
\text { DualPower TR, Pulse generator: BNC 575-8, Seeding particles generator: Flow } \\
\text { Visualization Components Flow Tracker 700CE }\end{array}$ \\
\hline $\begin{array}{c}\text { Time between } \\
\text { pulses, } \mu \mathrm{s}\end{array}$ & 56 \\
\hline Interrogation area & Size 32 x 32 (overlap 50\% in both directions), Central difference offset \\
\hline DPIV algorithm & Adaptive Correlation with Local Neighbourhood Validation \\
\hline Vector validation & Universal Outlier Detection method (normalized median validation) \\
\hline
\end{tabular}

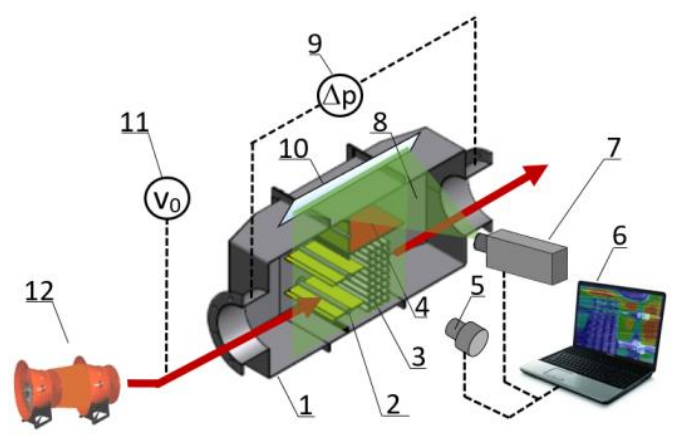

Fig. 2. Experimental stand and equipment: 1) transparent WHRU model, 2) main louver dampers, 3) tube bundle section, 4) bypass section, 5) CCD camera, 6) pulse generator and data acquisition, 7) double pulse Nd:YAG laser, 8) laser sheet, 9) gas differential manometer, 10) mirror,

11) thermo-anemometer, 12) fan unit.

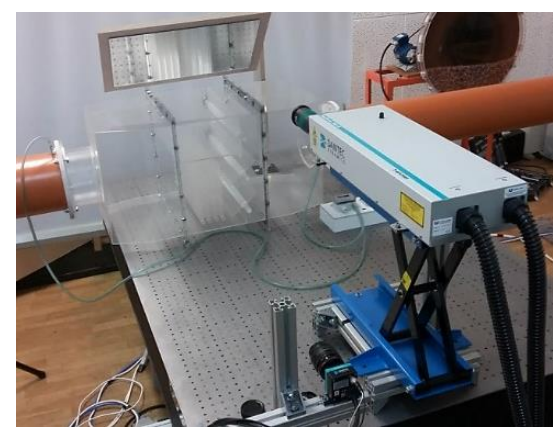

Fig. 3. View of experimental setup. 


\section{Results and discussions}

The main focus of the research reported here involved the determination of the velocity vector fields and streamlines, which make it possible to assess the velocity distribution and identification of vortex patterns in the WHRU. Due to the occurrence of the perspective phenomenon (covering the spacing between the tubes), a comprehensive analysis of the resulting visualizations was unfeasible. A similar problem was noted in the work [9]. However, the above optical effect did not apply to the region before the first vertical tube row, which became the basis for assessing the gas fraction. Considering the main mode of the operation of WHRU with full gas flow through a tube bundle section (position 1, fig. 4), flow maldistribution in the tube bundle section were recorded. At the level of the three horizontal lower tube rows, the forecasted value of the gas flow velocity derived from computations could not be achieved. An additional factor that negatively affected the performance of the device was associated with the occurrence of a maximum flow velocity of $5 \mathrm{~m} / \mathrm{s}$ only in the region located between the tube bundle and the baffle separating the tube bundle section from the bypass section. Despite the fact that the main louver dampers were completely opened, a vortex structure emerged directly behind the vertical structural element extending beyond the baffle separating both sections of the WHRU (Fig. 5a). In this operating mode, secondary air flows into the closed bypass section from the outlet side start to occur, which leads to the development of a vortex in the entire section volume. This phenomenon, in the case of the flow of a dusty agent, will lead to the deposition of contamination in the bypass section and the uncontrolled outflow when the section is opened.

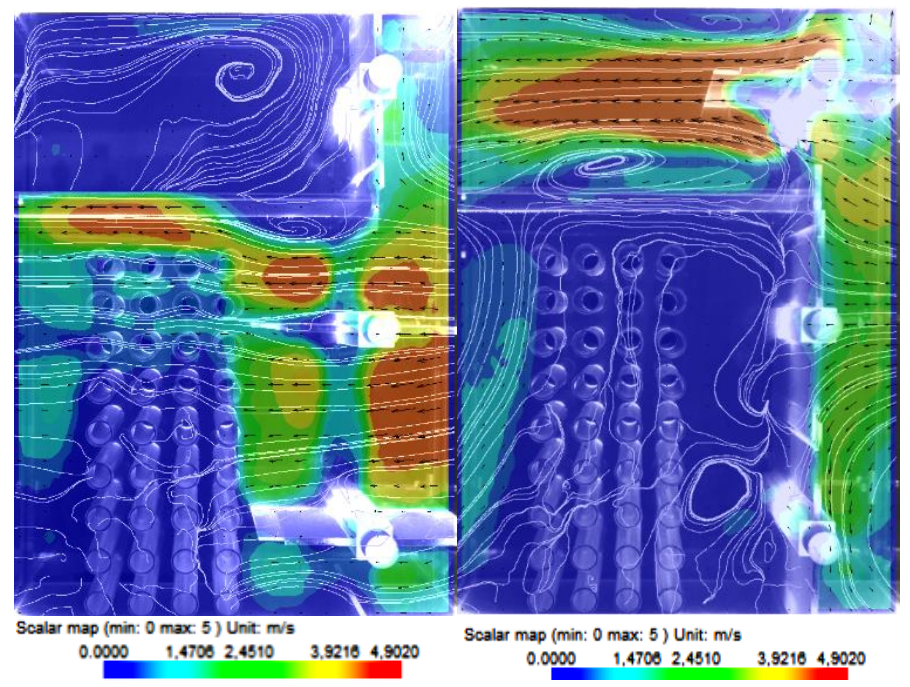

Fig. 4. Gas velocity vector field with a streamline in the WHRU during position 1 (on the left) and position 5 (on the right).

In the case of the analysis of the full gas flow through a bypass section, a similar relation was established between the occurrence of secondary air in this bypass (position 5 on Fig. 4). As a result of closing the main louver dampers, secondary air starts to flow into the tube bundle section. This is particularly undesirable due to potential contamination of the tube bundle and potential difficulties associated with removal of the resulting residue. In addition, leakage streams were identified as a consequence of the technological clearances. The total opening of the bypass louver damper with the main louver dampers closed also causes a vortex behind the extending structural element above baffle between the bypass section and 
the tube bundle section (Fig. 5b). Therefore, there is a need to redesign this element completely.
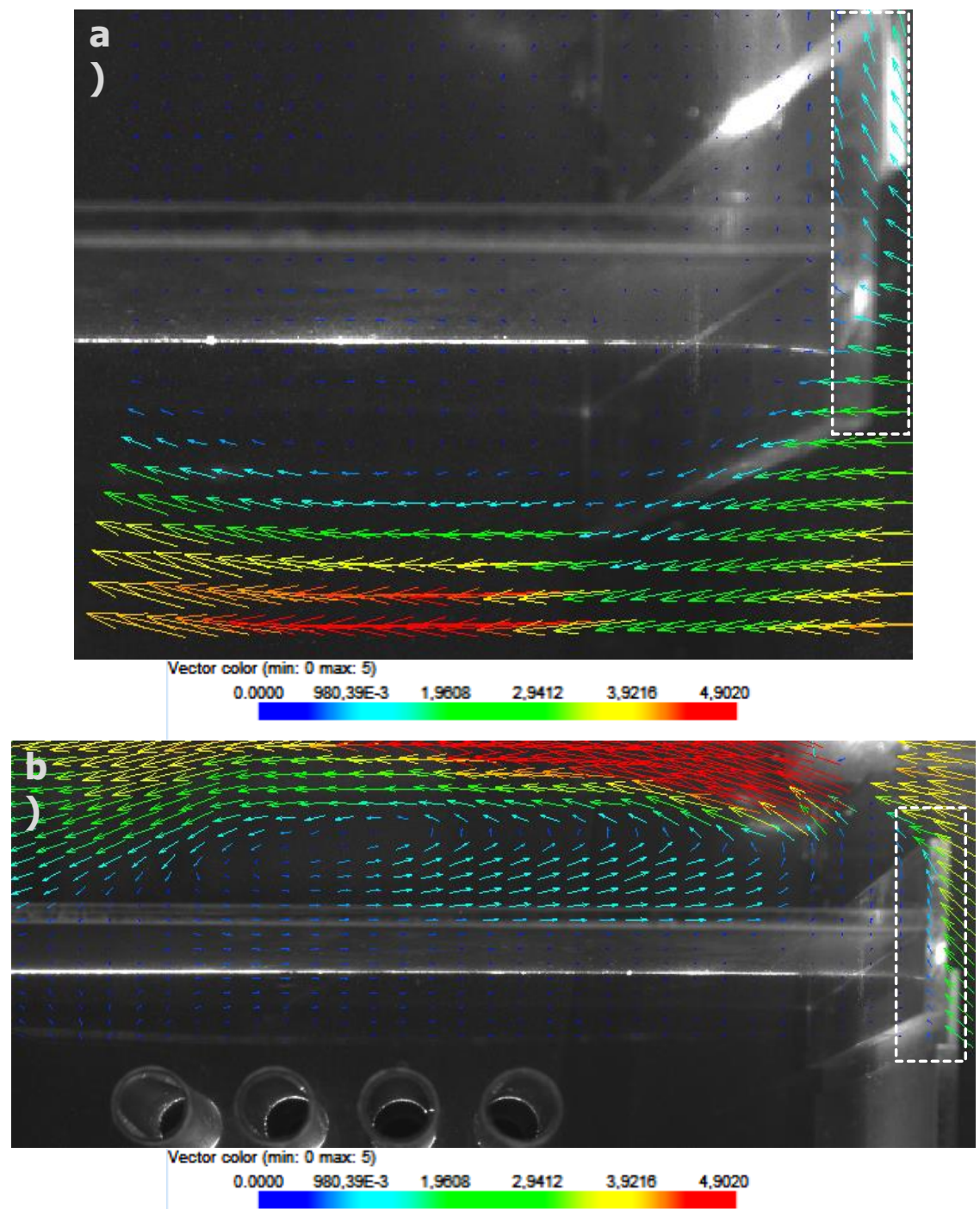

Fig. 5. The influence of the extending structural element (in a white frame) on the formation of vortex zones (vector velocity in $\mathrm{m} / \mathrm{s}$ ): a) position $1, \mathrm{~b}$ ) position 5 .

Figure 6 shows gas behaviour in transient states (positions 2, 3 and 4), which are primarily responsible for the direction of gas flow from the one section to another. The analysis of these operating modes is also particularly important because the control of the heat reception can be realized by selecting the appropriate configuration of louver dampers [16]. The combination of simultaneous gas flow in both sections and the blocking of gas flow through the upper main louver damper results in a decrease of the mean gas velocity in the tube bundle section. As a consequence, the amount of heat reception decreases as well. In positions 2, 3 and 4, maldistribution is intentional and expedient. The effect of these operating modes is associated with an increased intensity of the vortex phenomena. The vortex located behind the structural element extending beyond the baffle separating the two sections develops so as 
to occupy the space between this baffle and the upper main louver damper. In addition, an increase of the angle of main louver dampers leads to the development of another vortex region in the upper inlet slot to the tube bundle section. In turn, a huge vortex region is formed in the lower part of the bypass section irrespectively from the location of the bypass louver damper. It can be only reduced as a consequence of the full opening of the bypass section. The size of this vortex is also determined by the height of the structural element extending beyond the boundary of the partition between the sections. The limitation for the flow maldistribution observed in the above cases may be to relocate the inlet from the axis of symmetry of the front panel to the axis of symmetry of the tube bundle. Such operating mode should ensure an increase in the gas flow velocity. In such circumstances, gas flows directly into the tube bundle section without a negative effect of changes of the air flow direction and intensive vortices structures development.

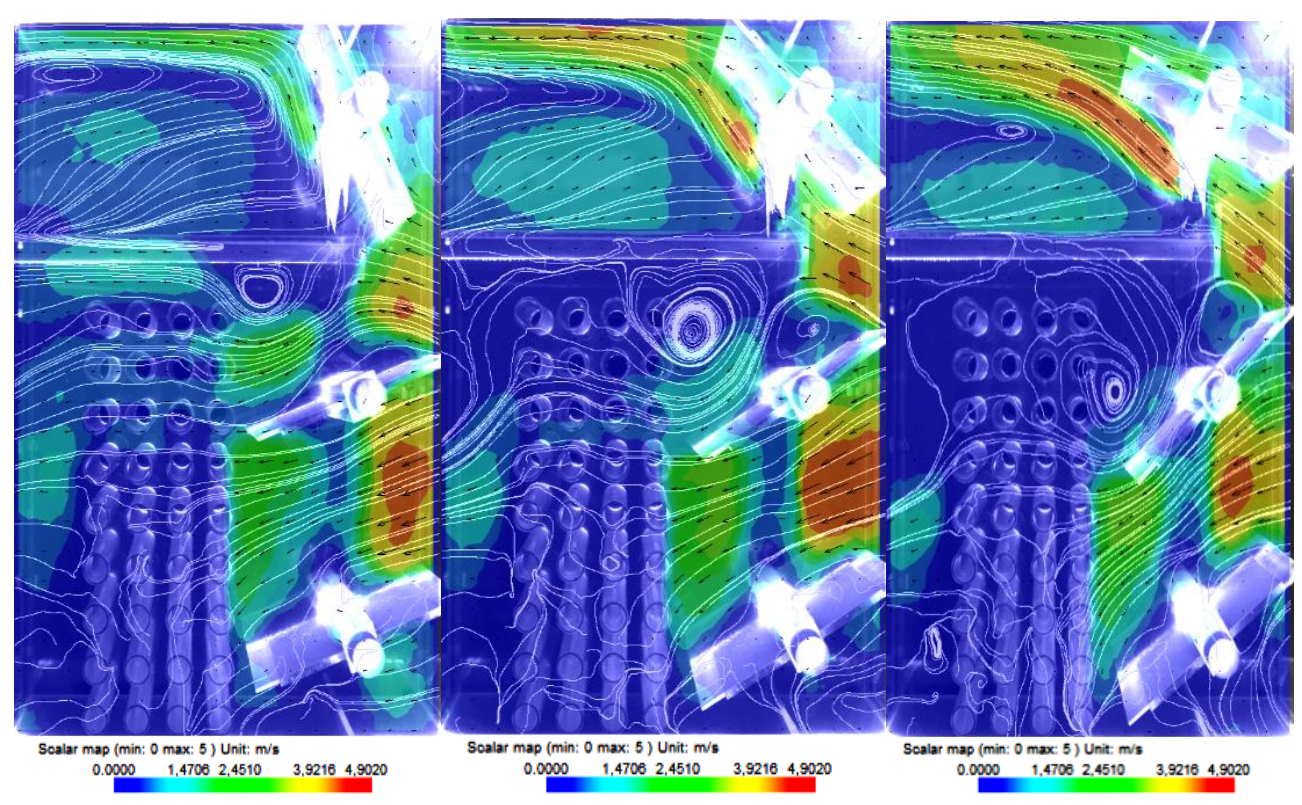

Fig. 6. Gas velocity vector field with a streamline in the WHRU during opening/closing the louver damper: position 2 (on the left), position 3 (in the middle) and position 4 (on the right).

A quantitative assessment was carried out into flow maldistribution depending on the configuration of louver dampers by comparing gas velocity profiles before the first vertical tube row (Fig. 7) for all tested locations. However, due to the fact that position 5 does not include the flow of the agent through the tube bundle, it is not taken into account in the quantitative analysis. The expected, calculated value of gas flow velocity $(4 \mathrm{~m} / \mathrm{s})$ was achieved only for position 1 and only at the height of the two upper tube rows. In contrast, at the height of the two bottom tube rows, the gas flow velocity greater than 0.5 of the calculated value could not be reached for any of the tested positions. By analyzing velocity vector fields, it was observed that along with an increase in the incline angle of the main louver dampers, the area of increased flow velocity is displaced downwards, at the expense of the top rows of tubes. On the basis of these observations, four regions of gas flow intensity in the tube bundle section were determined:

- $\quad$ high flow velocity zone $\left(\operatorname{Re}>2.02 \times 10^{5}\right)$

- medium flow velocity zone $\left(1.86 \times 10^{5}<\operatorname{Re}<2.02 \times 10^{5}\right)$

- low flow velocity zone $\left(\operatorname{Re}<1.86 \times 10^{5}\right)$ 
- the aerodynamic shadow region, which is characterized by a rapid drop of the velocity followed by a subsequent increase in its value. This region can occur irrespectively of the gas velocity.

In the case of positions 1 and 2, the zone in the height range of tube bundle section from $130 \mathrm{~mm}$ to $260 \mathrm{~mm}$ was identified. These areas are separated by a distinct aerodynamic shadow region developed by the upper main louver damper. The area of average gas velocity, that is characteristic of all tested positions, is located at a height range from $50 \mathrm{~mm}$ to $130 \mathrm{~mm}$ (except position 1, where due to the presence of the aerodynamic shadow zone, its lower limit must be raised up to $70 \mathrm{~mm}$ ). Below the height of tube bundle of $50 \mathrm{~mm}$, a low gas flow zone was determined for all positions. The occurrence of such a clear flow maldistribution in the tube bundle may create conditions for selective reception of the agent from particular horizontal rows of pipes, which leads to an improvement of the performance in terms of flexibility.

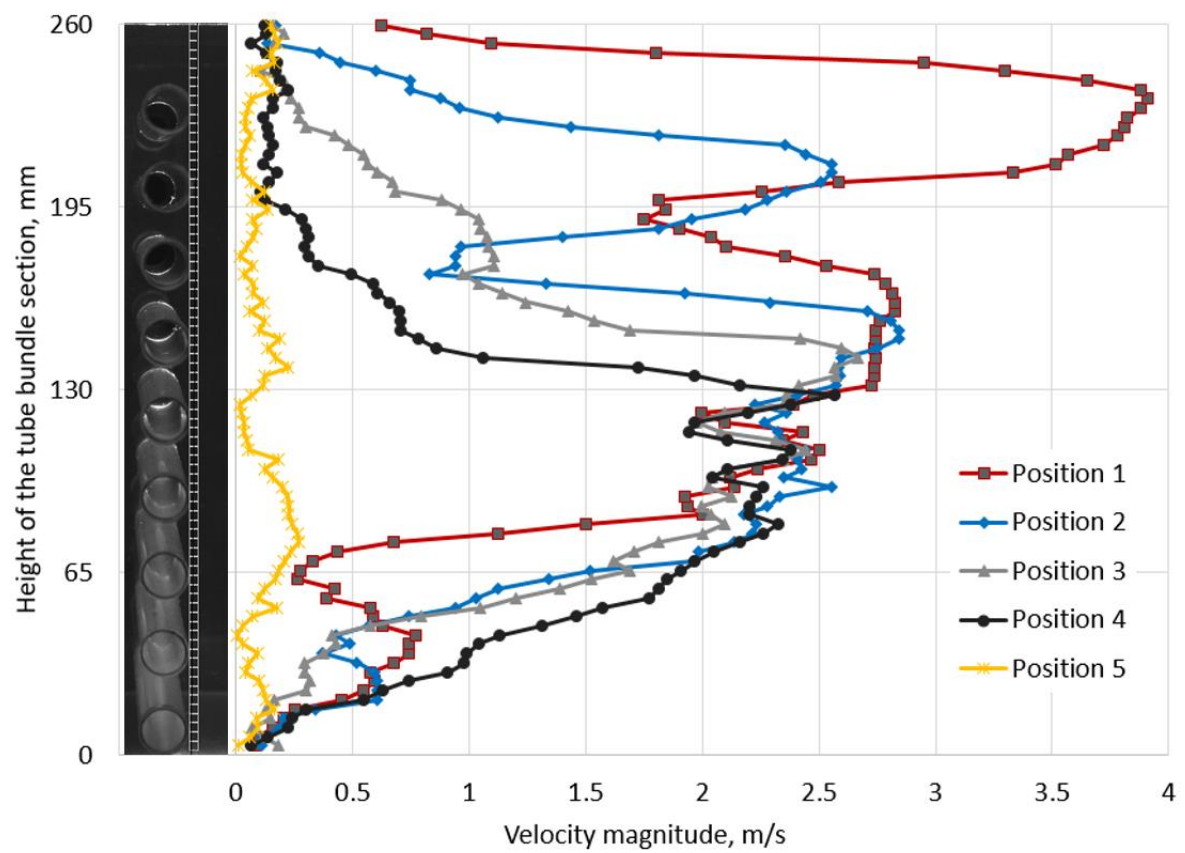

Fig. 7. Velocity magnitude profiles in the tube bundle section ahead of the first row of tubes with marked location of the measuring plane.

The uncertainty of the measurements of velocity vector fields depends on correctly recorded images of the phenomena. Assuming the correct type and size of the seeding particles as well as the optimal optical conditions of the equipment and identifying an average of 10 seeding particles in each interrogation area, the relative error of the DPIV method, as recommended by [10], was estimated to be equal to $10 \%$. To a lesser extent, the uncertainty of measuring is also affected by the uncertainty of estimating the displacement of seeding particles in the applied DPIV algorithm. Usually, it is in the range of 0.1-0.2 pixel unit [10]. According to the principle of displacement seeding particles by 0.25 linear dimension of the interrogation area, displacement at the level of 8 pixels was obtained. The studies in [11-13] determined that for these conditions the uncertainty in the measurement of seeding particles displacement is about $1-2 \%$. It is also necessary to take into account the occurrence of random uncertainty. On the basis of the study presented in [15], it was demonstrated in [14] that for the $95 \%$ confidence level (that is, a typical value that is assumed in experimental 
studies regarding gas phase flows), the random error could be determined in the range of 5\% of the mean velocity that is derived by the DPIV method.

\section{Conclusions}

The following conclusions can be stated on the basis of the experimental research into gas behavior in the heat exchanger applied for waste heat recovery:

- the DPIV method offers an effective diagnostic tool that enables the evaluation of the WHRU operating mode by reconstruction of flow structures and in particular the identification of vortices and maldistribution of gas,

- in order to eliminate partial maldistribution, it is recommended to reduce the distance of the tube bundle from the baffle that separates the tube bundle section and the bypass section,

- asymmetric location of the gas inlet relative to the tube bundle plays a significant role in the development of maldistribution of the gas. It is recommended to adjust the displacement of the gas inlet to a center position to be relative to the tube bundle,

- due to the fact that gas flow disturbance is generated, a suggestion is made to replace the vertical structural element extending beyond the baffle located between the sections of WHRU by application of another solution that can lead to stabilization of the louver dampers in the system.

\section{References}

1. M. Ghazi, P. Ahmadi, A. Sotoodeh, A. Taherkhani, Energy Convers. Manage. 58, 43-156 (2012)

2. F. Haug, J. Zheng, M. J. Beleynaud, J. Lu, J. Energy Inst. 90, 951-961 (2017)

3. M. Wasilewski, J. Duda, Powder Technol. 289, 143-158 (2016)

4. W. Ludwig, D. Zając, G. Ligus, P. Korman, Chem. Eng. Sci. 197, 386-403 (2019)

5. M. Wasilewski, Sep. Purif. Technol. 168, 114-123 (2016)

6. R. Goyal, B. K. Gandhi, M. J. Cervantes, Renew. Sust. Energ. Rev. 81, 2976-2991 (2018)

7. F. C. Li, K. Hishida, Adv. Chem. Eng. 37, 87-147 (2009)

8. P. Montgomery, Fundamentals of HVAC control systems (Elsevier, Amsterdam, 2008)

9. I. Sunghyuk, T..K. Hyoung, W..R. Bo, J..S. Hyung, Ann. Nucl. Energy 98, 1-11 (2016)

10. R. J. Adrian, J. Westerweel, Particle Image Velocymetry (Cambridge University Press, New York, 2011)

11. C. E. Willert, Appl. Sci. Res. 56, 79-102 (1996)

12. J. M. Foucaut, B. Miliat, N. Perenne, M. Stanislas, Particle Image Velocimetry: Recent Improvements (Springer, Berlin, 2008)

13. J. Westerweel, Exp. Fluids 26, 3-12 (2006)

14. B. P. Husted, P. Petersson, I. Lund, G. Holmstedt, Fire Safety J. 44, 1030-1045 (2009)

15. H. W. Coleman, W. G. Steele, Experimentation and Uncertainty Analysis for Engineers (John Wiley \& Sons, New York, 1999)

16. V. Vinod, S. Sathishkumar, R. K. Mourya, S. Chandramouli, G. Padmakumar, B. K. Nashine, P. Selvaraj, Nucl. Eng. Des. 318, 174-181 (2017) 